\title{
Interventions sur les tubes-guides de grappes de contrôle des réacteurs à eau préssurisée (REP 900 MW)
}

\author{
R. DOLLO* \\ (Manuscrit reçu le 9 mai 1984)
}

\begin{abstract}
RÉSUME
Des incidents successifs sur quatre tranches REP $900 \mathrm{MW}$ ont affectés en 1982 les broches de centrage des tubes-guides de contrôle. Ces incidents ont conduit dans un premier temps à la mise au point de contrôles télévisuels, puis non destructifs, et enfin à la décision de remplacer en deux ans toutes les broches de l'ensemble des tranches françaises en fonctionnement au moment des incidents, soit 21 tranches. Les broches des tranches en construction ont été remplacées avant démarrage à partir de celle de Blayais 2.
\end{abstract}

Cet article présente la synthèse des différents coûts dosimétriques des interventions sur les tubes-guides.

\section{ABSTRACT}

Four $900 \mathrm{MW}$ standard PWR units were affected successively in 1982 by flaws in control-rod drive mechanisms (CRDM). These flaws first required developing TV inspection methods, followed by non-destructive tests, and finally the replacement of the complete sets of CRDMs on all the French units operating at that time (21 units). CRDMs on units still in construction were replaced, beginning with the Blayais 2 unit.

The global results are given in terms of doses associated with work on CRDMs.

\section{INTRODUCTION}

La découverte d'une lame flexible d'une broche d'un tube-guide dans un clapet de la tranche 1 de Gravelines en janvier 1982, suivie d'incidents sur d'autres tranches, a mis en évidence le caractère générique du problème. Des incidents analogues avaient eu lieu dans les centrales japonaises (en 1978 et 1979) et aux USA (fissures Ohi, 1980 ; cassures North Anna, 1982). L'expertise menée par les exploitants français a confirmé celles réalisées par les experts étrangers, qui indiquaient que les broches étaient rompues par un phénomène de corrosion sous tension.

- Electricité de France, Service de la production thermique, Département Sécurité, Radioprotection, Environnement, 6, rue Ampère, BP 114, 93203 Saint-Denis Cedex 1. 
Les incidents de broches survenus successivement sur plusieurs tranches françaises ont conduit le Service de la production thermique d'EdF à prendre des mesures en collaboration avec la société Framatome et à mettre en œuvre les moyens, tant matériels qu'humains, nécessaires pour faire face à la situation afin de préparer une stratégie globale de réparation. Cette stratégie s'est appliquée de la façon suivante :

a) Sur la tranche 1 de Gravelines, vérification avant démarrage à l'aide d'un contrôle télévisuel de la présence de deux lames de chaque broche des tubes-guides ;

b) Examen par le laboratoire chaud de Chinon (SCMI) ${ }^{(1)}$ de la lame flexible en vue de procéder à des contrôles métallurgiques poussés pour comprendre son mauvais comportement; des études et des essais ont également été entrepris pour améliorer la tenue des broches sur le plan métallurgique et sur le plan mécanique ;

c) Etude de sûreté par analyse des risques liés à la rupture d'une broche ; cette analyse a conduit à l'étude de deux types de risque :

- risques liés aux corps migrants que peuvent constituer les écrous ou les branches flexibles,

- risques liés à la perte de fonction de guidage des tubes-guides aussi bien en fonctionnement normal qu'en fonctionnement accidentel ; cette étude de sûreté a abouti, en avril 1983, à la conclusion que l'endommagement des broches n'entraîne pas de conséquences préjudiciables au plan de la sûreté de l'installation.

L'expérience japonaise laissait craindre les mêmes incidents sur les tranches françaises ; aussi, dès 1980, Framatome a mis à l'étude un appareil d'examen ultra-sonore des broches et l'outillage de changement des broches.

Pour les réacteurs en construction, des améliorations recommandées par le constructeur ont été retenues pour diminuer les contraintes de serrage et pour optimiser les traitements thermiques. Ces modifications ont été introduites progressivement dans les tranches françaises, ce qui a conduit à classer les broches en plusieurs familles.

Pour la première famille, le remplacement total des broches s'est imposé immédiatement. Sur toutes les autres tranches suspectes, le changement des broches s'est engagé de façon à étaler cette opération dans le temps, l'objectif étant de profiter des arrêts de tranches programmés au changement de combustible pour réaliser autant que possible le travail en "temps masqué".

Des dispositions complémentaires ont été prises telles que le contrôle du temps de chute des grappes de contrôle, et une surveillance acoustique renforçée.

\section{PRESENTATION DU PROBLEME TECHNIQUE - ROLE DES PIECES INCRIMINEES}

Dans les réacteurs REP de conception Westinghouse, le combustible nucléaire est maintenu en place dans la cuve par l'intermédiaire d'une

(1) Service de contrôle des matériaux irradiés, dépendant du Service de la production thermique. 
structure complexe appelée interne supérieure. Au-dessus des éléments combustibles recevant des grappes de contrôle, des tubes servant au guidage des grappes, au nombre de 61 pour les tranches du premier palier, sont fixés dans cette structure (fig. 1). Ces tubes-guides sont suspendus en partie supérieure à la plaque support des tubes-guides. Afin d'éviter les déplacements latéraux tout en laissant libres les déplacements verticaux permettant ainsi les dilatations différentielles entre les tubesguides et les colonnes entretoises des structures internes supérieures, ils sont guidés, en partie inférieure, par 2 broches (fig. 2).

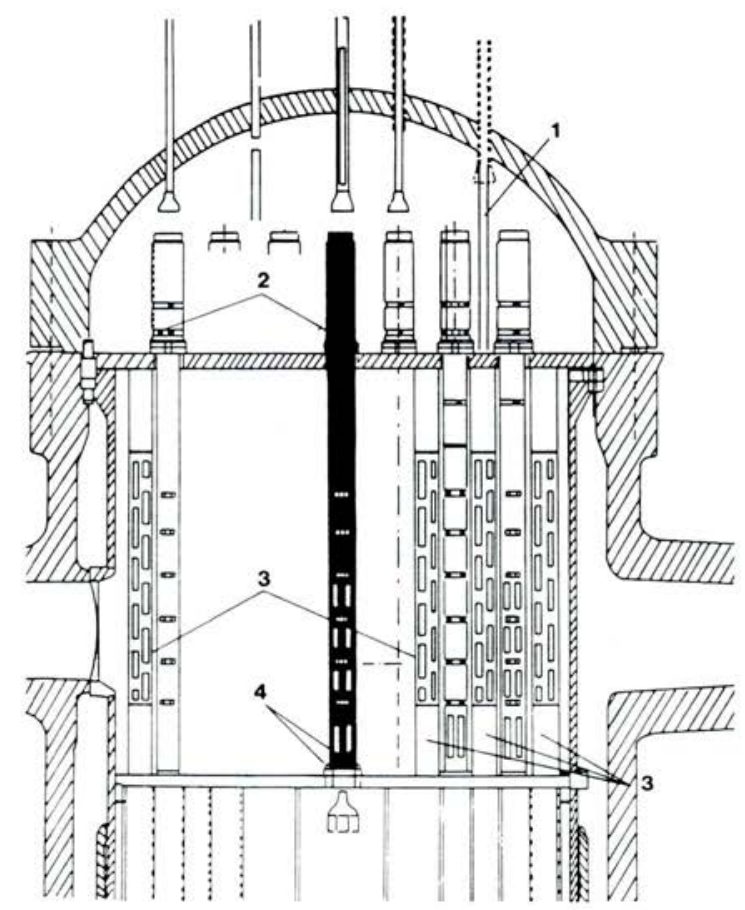

1. Colonnes thermo-couples.

2. 4 vis de fixation sur plaque de support.

3. Colonnes entretoises.

4. 2 broches de guidage.

Fig. 1. - Internes supérieurs en cuve. 


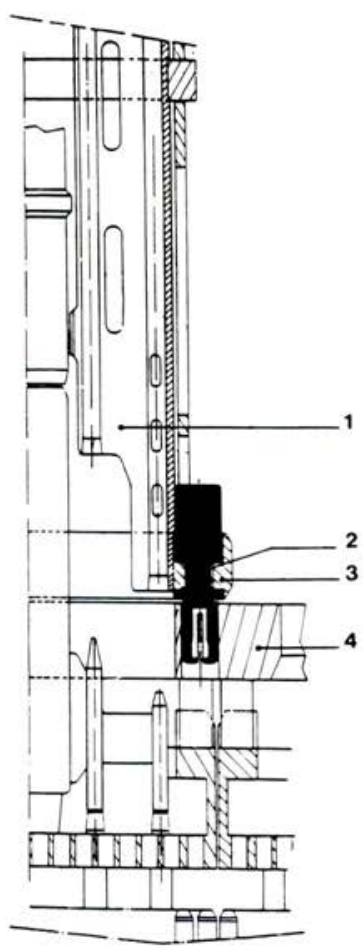

1. Tube-guide de grappe.

2. Broche.

3. Bride inférieure du guide de grappe.

4. Plaque supérieure du cœur.

Fig. 2. - Emplacement de la broche.

\section{Rôle et caractéristiques des broches}

Ces broches doivent résister aux différents efforts transmis par les tubes-guides : efforts permanents (trainée) et vibratoires. Elles doivent aussi assurer une liaison glissante sans jeu avec la plaque supérieure du cœur pour maintenir l'alignement des tubes-guides et des gaines réservées dans le combustible. Pour remplir ces fonctions, les broches sont fixées grâce à leur extrémité filetée et un écrou sur la bride inférieure du tube-guide. L'extrémité inférieure fendue, pour constituer deux lames élastiques, pénètre dans un trou pratiqué dans la plaque supérieure du cœur. Ces deux lames sont précontraintes au montage (fig. 3). Les efforts importants à reprendre et la dimension très limitée de ces pièces ont conduit à retenir comme matériau l'Inconel $\times 750$, alliage inoxydable à hautes caractéristiques mécaniques, apparu comme le plus apte à répondre aux exigences demandées. 


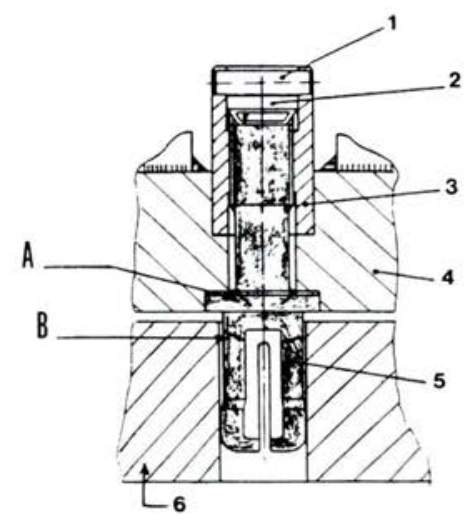

1. Barrette.

2. Chapeau d'arrêt.

3. Ecrou.

4. Bride inférieure du guide de grappe.

5. Broche fendue.

6. Plaque supérieure du cœur.

A. Zone de fissure $A$.

B. Zone de fissure $B$.

Fig. 3. - Coupe de la broche (hauteur: $10 \mathrm{~cm}$, diamètre: $2 \mathrm{~cm}$ ).

\section{PREMIERS INCIDENTS}

En janvier 1982, à Gravelines 1, une lame flexible d'une broche s'est cassée après $10000 \mathrm{~h}$ de fonctionnement du réacteur.

En mars 1982, à Fessenheim 1, une broche s'est cassée au-dessus du collet après $30000 \mathrm{~h}$ de fonctionnement du réacteur. L'écrou de la broche a entraîné un matage important des extrémités des tubes de la boîte à eau du générateur de vapeur $n^{\circ} 3$.

Fin juillet 1982, à Bugey 2, un incident analogue à celui de Fessenheim est survenu : une broche s'est cassée après $25000 \mathrm{~h}$ de fonctionnement du réacteur, et son écrou a entraîné un léger martelage des tubes d'un générateur de vapeur. 
R. DOLLO

IV. CHRONOLOGIE DES OPERATIONS

TABLEAU I

\begin{tabular}{|c|c|c|}
\hline Tranches & Dates & Descriptions " succinctes" \\
\hline \multirow[t]{5}{*}{ Fessenheim 1} & 19.07 .82 & . $1^{\text {re }}$ intervention sur 2 tubes-guides ; \\
\hline & 20.10 .82 & mise en place de tubes-guides neufs \\
\hline & & outillage dit " bas de gamme" ; \\
\hline & & plate-forme à la cote $13 \mathrm{~m}$ \\
\hline & & $\begin{array}{l}\text { évacuation tube-guide en air sous hotte vers } \\
\text { piscine du bâtiment combustible. }\end{array}$ \\
\hline \multirow[t]{4}{*}{ Bugey 4} & 25.09 .82 & mise en place de tubes-guides neufs ; \\
\hline & 08.11 .82 & plate-forme à la cote $16 \mathrm{~m}$ \\
\hline & & outillage " haut de gamme" ; \\
\hline & & $\begin{array}{l}\text { évacuation tube-guide sous eau par tube de } \\
\text { transfert. }\end{array}$ \\
\hline \multirow[t]{2}{*}{ Bugey 2} & 20.10 .82 & mise en place de tubes-guides neufs ; \\
\hline & 17.11 .82 & outillage moins sophistiqué. \\
\hline \multirow[t]{2}{*}{ Bugey 3} & 09.02 .83 & changement de 3 tubes-guides par des neufs. \\
\hline & 18.02 .83 & \\
\hline \multirow[t]{3}{*}{ Fessenheim 2} & 15.02 .83 & conservation des tubes-guides ; \\
\hline & 19.03 .83 & remplacement des broches ; \\
\hline & & $\begin{array}{l}\text { utilisation d'un stand fabriqué par la centrale } \\
\text { appelé "Totem". }\end{array}$ \\
\hline \multirow[t]{4}{*}{ Tricastin 1} & $\begin{array}{l}31.01 .83 \\
21.03 .83\end{array}$ & $\begin{array}{l}\text { initialement changement de broches par } \\
\text { stands chauds Framatome ; }\end{array}$ \\
\hline & & stands non opérationnels ; \\
\hline & & $\begin{array}{l}\text { décision d'un changement des tubes-guides } \\
\text { par récupération des tubes-guides neufs de } \\
\text { Cruas 2 : }\end{array}$ \\
\hline & & $\begin{array}{l}\text { évacuation tube-guide en air sous hotte vers } \\
\text { piscine du bâtiment combustible. }\end{array}$ \\
\hline Bugey 5 & $\begin{array}{l}09.05 .83 \\
29.05 .83\end{array}$ & $\begin{array}{l}\text { changement des } 53 \text { tubes-guides par des } \\
\text { tubes-guides préalablement réparés pro- } \\
\text { venant des EIS (élements internes supérieurs) } \\
\text { de Bugey } 2 \text { et } 4 \text { (temps masqué méthode } \\
\text { Totem + stand chaud simplifié). }\end{array}$ \\
\hline Graveline 1 & $\begin{array}{l}11.05 .83 \\
04.06 .83\end{array}$ & $\begin{array}{l}\text { changement des tubes-guides par ceux de } \\
\text { Tricastin } 1 \text { préalablement réparés à Tricastin } \\
\text { (Usines à broches + stand chaud simplifié). }\end{array}$ \\
\hline
\end{tabular}




\begin{tabular}{|c|c|c|}
\hline Tranches & Dates & Descriptions " succinctes" \\
\hline \multirow[t]{3}{*}{ Tricastin 2} & $\begin{array}{l}23.05 .83 \\
05.07 .83\end{array}$ & $\begin{array}{l}\text { remplacement de tubes-guides par le jeu } \\
\text { de tubes-guides de Gravelines } 1\end{array}$ \\
\hline & & $\begin{array}{l}\text { méthode "Aquarium" mise au point conjoin- } \\
\text { tement par la centrale de Tricastin et l'unité } \\
\text { technique opérationnelle : }\end{array}$ \\
\hline & 06.83 & $\begin{array}{l}\text { création sur le site de Pierrelatte d'un atelier } \\
\text { "changement de broches" avec utilisation } \\
\text { de } 4 \text { stands chauds Framatome (atelier B). }\end{array}$ \\
\hline \multirow[t]{5}{*}{ Tricastin 4} & $\begin{array}{l}15.10 .83 \\
03.12 .83\end{array}$ & $\begin{array}{l}\text { remplacement des tubes-guides par ceux } \\
\text { provenant de Tricastin } 2 \text {; }\end{array}$ \\
\hline & & opération réalisée sur le site ; \\
\hline & & . utilisation de la méthode dite "Aquarium". \\
\hline & 05.12 .83 & $\begin{array}{l}\text { mise en exploitation de l'atelier à broches } \\
\text { de Pierrelatte. }\end{array}$ \\
\hline & 26.06 .84 & $\begin{array}{l}\text { changement des broches de tubes-guides } \\
\text { provenant de Tricastin } 4 \text {, ceux non utilisés } \\
\text { de Tricastin } 2 \text {, Bugey } 5 \text {, Dampierre 1, Bugey } 3 \text {, } \\
\text { Gravelines } 4 \text {. }\end{array}$ \\
\hline \multirow[t]{2}{*}{ Dampierre 1} & $\begin{array}{l}03.02 .84 \\
06.04 .84\end{array}$ & $\begin{array}{l}\text { remplacement des tubes-guides par le jeu } \\
\text { de Tricastin } 4 \text { équipé de nouvelles broches } \\
\text { mises en place à l'atelier B }\end{array}$ \\
\hline & & $\begin{array}{l}\text { permutation des tubes-guides à l'aide de } \\
\text { conteneurs et d'une hotte de manutention. }\end{array}$ \\
\hline \multirow[t]{2}{*}{ Bugey 3} & $\begin{array}{l}09.03 .84 \\
30.04 .84\end{array}$ & $\begin{array}{l}\text { remplacement des tubes-guides par ceux } \\
\text { de Bugey } 5 \text { équipés de broches neuves ; }\end{array}$ \\
\hline & & $\begin{array}{l}\text { permutation des tubes-guides par la méthode } \\
\text { dite "conteneurs". }\end{array}$ \\
\hline \multirow[t]{2}{*}{ Gravelines 4} & $\begin{array}{l}25.04 .84 \\
02.06 .84\end{array}$ & $\begin{array}{l}\text { utilisation du jeu de tubes-guides de Dampierre } 1 \\
\text { après équipement à l'atelier B }\end{array}$ \\
\hline & & $\begin{array}{l}\text { "changement des tubes-guides par la méthode } \\
\text { "Aquarium". }\end{array}$ \\
\hline \multirow[t]{2}{*}{ Dampierre 2} & $\begin{array}{l}16.06 .84 \\
12.08 .84\end{array}$ & $\begin{array}{l}\text { utilisation des tubes-guides provenant de } \\
\text { Bugey } 2 \text { \& } 3 \text { après passage à l'atelier B }\end{array}$ \\
\hline & & $\begin{array}{l}\text { "changement des tubes-guides par la méthode } \\
\text { "conteneurs". }\end{array}$ \\
\hline \multirow[t]{2}{*}{ Gravelines 3} & $\begin{array}{l}01.08 .84 \\
08.09 .84\end{array}$ & $\begin{array}{l}\text { utilisation des tubes-guides provenant de } \\
\text { Gravelines } 4 \text { après équipement de broches } \\
\text { neuves à l'atelier B: }\end{array}$ \\
\hline & & $\begin{array}{l}\text { "permutation des tubes-guides par la méthode } \\
\text { "Aquarium". }\end{array}$ \\
\hline
\end{tabular}




\section{DOSIMETRIE COLLECTIVE}

\section{Résultats globaux par tranche}

TABLEAU ॥

Dosimétrie collective (en $\mathrm{mSv}$ )

$\begin{array}{ll}\text { Fessenheim 1 } & : 503 \\ \text { Bugey 4 } & : 200 \\ \text { Bugey 2 } & : 85 \\ \text { Bugey 3 } & : 10 \text { (non représentatif changement 3 tubes-guides) } \\ \text { Fessenheim 2 } & : 152 \\ \text { Tricastin 1 } & : 306 \\ \text { Bugey 5 } & : 385 \\ \text { Gravelines 1 } & : 394 \\ \text { Tricastin 2 } & : 158 \\ \text { Tricastin 4 } & : 138 \\ \text { Dampierre 1 } & : 103 \\ \text { Bugey 3 } & : 255 \\ \text { Gravelines 4 } & : 254 \\ \text { Dampierre 2 } & \end{array}$

Afin d'analyser ces résultats, il s'avère intéressant de classer les opérations en 4 types:

A. Mise en place de tubes-guides neufs concernant les tranches de Fessenheim 1, Bugey 4, Bugey 2, Tricastin 1 ;

B. Conservation des tubes-guides irradiés, changement des broches sur site par méthode "Totem" et stand chaud Framatome (lorsqu'il est disponible) concernant Fessenheim 2 et Bugey 5 ;

C. Utilisation de l'usine à broches de Tricastin, récupération de jeux de tubes-guides irradiés d'autres centrales (stand chaud simplifié) : Gravelines 1, Tricastin 2, Tricastin 4 ;

D. Utilisation de l'usine à broches de Pierrelatte avec stands chauds.

Les tubes-guides deviennent banalisés sur l'ensemble du palier $900 \mathrm{MW}$. Le changement des broches se déroule par campagne; le nombre de tubes-guides concernés à chaque campagne varie selon qu'ils sont conservés ou remplacés par des tubes-guides neufs ou irradiés en provenance d'une autre centrale. Dans ce cas, la dosimétrie pour le changement de broches est donnée dans le tableau III.

TABLEAU III

\begin{tabular}{|l|c|r|}
\hline \multicolumn{1}{|c|}{$\begin{array}{c}\text { Provenance } \\
\text { des tubes-guides }\end{array}$} & $\begin{array}{c}\text { Nombre de } \\
\text { tubes-guides }\end{array}$ & Dose en mSv \\
\hline Tricastin 4 & 32 & 52 \\
Tricastin 1, 2, 4, Bugey 5 & 49 & 39 \\
Dampierre 1 & 62 & 101 \\
Bugey 3 & 32 & 19 \\
Gravelines 4 & 61 & 36 \\
\hline
\end{tabular}




\section{Analyse détaillée par type d'opération}

\subsection{Opération de type A (Fessenheim 1, Bugey 4, Bugey 2, Tricastin 1)}

Du fait qu'il fallait réagir rapidement, l'ensemble des tubes-guides-broches, sur les 4 tranches considérées (voir tableau II), a été remplacé par des ensembles neufs.

L'analyse de ce bilan dosimétrique fait apparaître une exposition plus élevée pour les centrales de Fessenheim et de Tricastin que pour celle de Bugey. Pour mieux connaître les raisons, il est nécessaire de développer les points suivants : l'intervention à Fessenheim sur deux tubesguides pour essais était une première ; les méthodes d'intervention étaient différentes entre les centrales; une vis grippée à nécessité une opération particulière à Fessenheim ; la méthode d'intervention a été changée à Tricastin, les stands chauds n'étant pas opérationnels.

\section{a) Essais}

La centrale de Fessenheim a dû extraire deux tubes-guides à titre d'essai. Cette première intervention relativement longue (4 à 5 jours) a permis de s'assurer de la faisabilité de la méthode. Sur le plan de la radioprotection, elle a mis en évidence l'existence d'un débit de dose élevé dû à la présence des colonnes thermo-couples. Pour limiter les rayonnements, il a fallu renforcer la protection biologique existante (coût de cette première intervention: $70 \mathrm{mSv}$ ).

\section{b) Méthodes}

La méthode consiste à déposer les internes supérieures sur leur stand, puis à extraire les tiges de commande et à les stocker dans des étuis regroupés dans un rack placé dans la piscine. D'une plate-forme installée au-dessus des internes, les opérateurs introduisent à l'intérieur du tube-guide un grappin ouvert en partie basse à la manière d'un parapluie, ce qui permet d'extraire le tube-guide en le poussant par le bas. Lorsque l'ensemble des tubes-guides est extrait, après contrôle des plaques supports, des tubes-guides neufs équipés d'une nouvelle génération de broches sont remontés (fig. 4).

Les centrales de Bugey et de Tricastin, grâce à l'utilisation d'un outillage performant ont travaillé en permanence avec une hauteur d'eau suffisante (niveau cote : $16 \mathrm{~m}$ ) ; par contre, la centrale de Fessenheim, dotée d'un outillage moins performant, est intervenue avec un niveau d'eau dans la piscine-réacteur moins élevé (cote : $13 \mathrm{~m}$ ). Cette différence de l'ordre de $3 \mathrm{~m}$ augmente sensiblement la valeur du débit de dose d'ambiance. De fait, le débit de dose d'ambiance autour de la plate-forme est très influencé par la présence du rack de stockage des tiges de commande des barres de contrôle et des colonnes de thermocouples. A Fessenheim, les tiges étaient dénoyées de $2 \mathrm{~m}$.

Valeurs moyennes d'ambiance autour de la plate-forme :

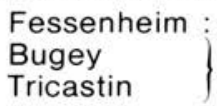

0,1 à $0,5 \mathrm{mSv} / \mathrm{h}$

Tricastin

0,02 à $0,1 \mathrm{mSv} / \mathrm{h}$. 


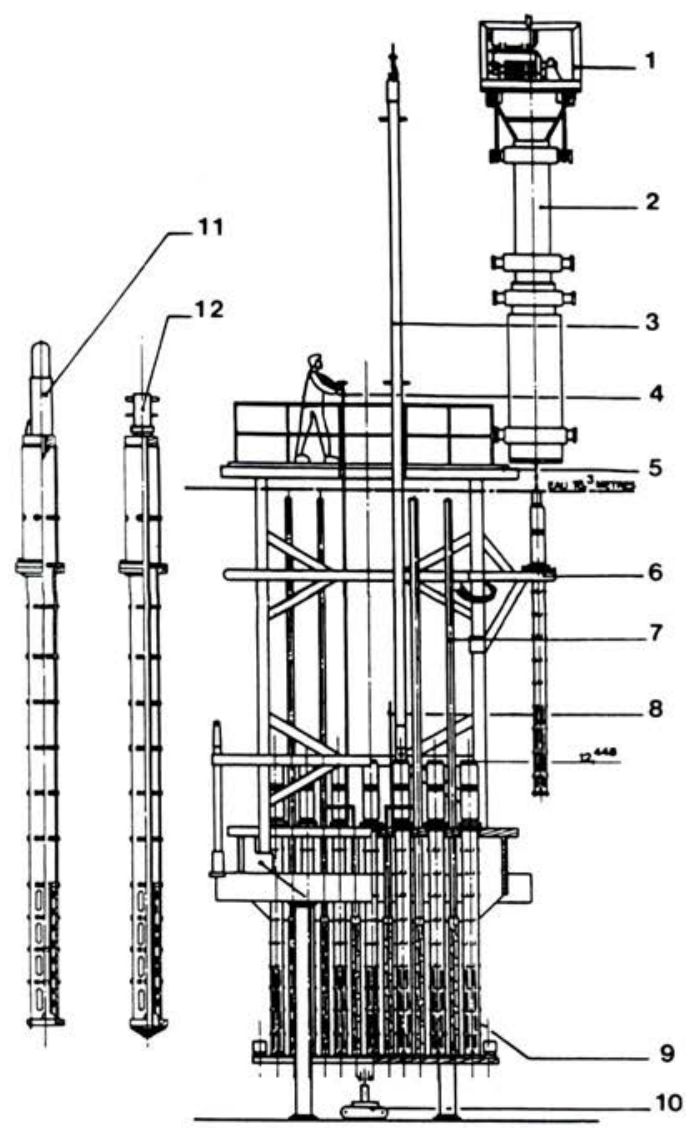

1. Palonnier.

2. Hotte.

3. Outil de manutention long du grappin.

4. Outil de vissage.

5. Plate-forme $16,6 \mathrm{~m}$.

6. Potence pivotante.
7. Colonne thermo-couple.

8. Tige de guidage.

9. Rehausse.

10. Chariot inspectronic "Oscar".

11. Grappin à cliquets.

12. Grappin parapluie.

Fig. 4. - Plate-forme de travail.

Pour les interventions ultérieures, le rack de stockage a été placé dans la piscine, côté réacteur.

L'extraction et l'évacuation des tubes-guides vers la piscine du bâtiment combustible ont été réalisées à Fessenheim et à Tricastin par l'intermédiaire d'une hotte en plomb. Bugey a réalisé ces opérations sous eau en utilisant le tube transfert modifié pour la circonstance. 


\section{c) Opération particulière (Fessenheim)}

Au cours du démontage d'un tube-guide, on a constaté le grippage d'une vis. La nécessité de mettre au point une autre technique (de type perçage, alésage, récupération des copeaux...) s'est avérée coûteuse en dose. Cette opération s'est traduite par un coût dosimétrique élevé de l'ordre de $70 \mathrm{mSv}$ dû, en partie, à une improvisation du matériel à utiliser.

\section{d) Changement de méthode}

A Tricastin, le non fonctionnement des stands chauds a demandé une transformation complète du scénario initial, consistant à mettre en place des tubes-guides neufs provenant de Cruas 2 . II a fallu, en 5 jours, récupérer et mettre en place des matériels permettant le transfert des tubes-guides et leur stockage dans le bâtiment combustible. L'opération d'évacuation n'a pas pu se réaliser sous eau comme à la centrale de Bugey, car les transformations du tube de transfert demandaient trop de temps. Cette manutention importante a été réalisée à l'aide de 2 hottes et de 6 personnes.

Le chantier de Fessenheim a apporté de nombreux enseignements et les centrales de Bugey et de Tricastin ont su utiliser cette expérience pour améliorer les conditions d'intervention. Le facteur durée d'intervention est important : par exemple, la durée du chantier Bugey 2 a été plus courte de 10 jours par rapport à celle de Bugey 4 : l'utilisation d'un outillage trop sophistiqué sur Bugey 4 a été à l'origine de nombreuses défaillances, se répercutant sur la durée des interventions et les coûts dosimétriques associés. Dans ce domaine, il faut rechercher la simplicité.

Enfin, tout changement impromptu dans les méthodes ou l'outillage utilisé est un facteur d'augmentatin de la dose (cas de Tricastin) ; comme nous le verrons dans la suite des opérations, la réduction des doses exige des procédures bien "rodées" et un personnel bien entraîné.

\subsection{Opération de type $B$ (Fessenheim 2, Bugey 5)}

A la suite de la rupture des broches de tubes-guides sur différentes tranches et compte tenu de l'aspect générique du problème, il a été décidé de procéder au remplacement de toutes les broches sur le site et sur l'ensemble des tubes-guides avec réutilisation de tubes-guides irradiés appartenant à la tranche concernée - pour Fessenheim, et des tranches 2 et 4 de Bugey pour la tranche Bugey 5. L'opération devait s'effectuer au moyen d'un stand chaud développé par Framatome. Ce stand très sophistiqué demandait de nombreuses mises au point; aussi, en accord avec Framatome, un stand de secours a été fabriqué à la centrale de Fessenheim. Seules, les soudures des broches ont été réalisées sur stand chaud.

Les opérations de remplacement des broches et de manutention des tubes-guides ont été effectuées sur des matériels fortement irradiés. Des interventions directes ont eu lieu d'une part sur les broches situées en partie inférieure du tube-guide (débit de dose au contact compris entre 5 et $7 \mathrm{~Sv} / \mathrm{h}$ ) et d'autre part, pour le remplacement des coupelles, au niveau 
des brides de liaison des parties inférieure et supérieure des tubes-guides (débit de dose de l'ordre de 20 à $30 \mathrm{mSv} / \mathrm{h}$ ). L'utilisation de la hotte de transport et la mise en place de différentes protections biologiques ont permis de diminuer considérablement le débit de dose, mais l'accès aux pièces à remplacer devait se faire directement par des fenêtres avec un matériel "rustique".

Des difficultés liées au manque d'expérience des opérateurs au début d'intervention et à la mise au point de l'outillage ont conduit inévitablement à un bilan dosimétrique élevé pour la centrale de Fessenheim.

Cette expérience d'utilisation de la méthode dite "Totem" sera profitable aux tranches suivantes: Bugey 5 et Tricastin 2. En effet, l'examen du bilan dosimétrique de Bugey 5 met en évidence les bénéfices de l'expérience acquise (chantier Totem + stand chaud "allégé" coût : $59 \mathrm{mSv}$ ). Malheureusement, ce gain est remis en cause par une opération très pénalisante, à savoir le transfert des tubes-guides (Bugey 2, 3, 4) vers un bâtiment affecté provisoirement aux changements de broches (coût : $193 \mathrm{mSv}$ ). Une protection biologique (mince écran d'eau) mal adaptée et située à la partie supérieure du conteneur entraînait un débit de dose de l'ordre de $3 \mathrm{mSv} / \mathrm{h}$. On a cherché à améliorer cette protection en augmentant la hauteur d'eau, mais un problème d'étanchéité n'a jamais pu être résolu.

Grâce à l'expérience acquise, ces conteneurs ont été par la suite améliorés, notamment par l'adjonction d'une protection biologique complémentaire extérieure et d'une garde assurant une hauteur d'eau supplémentaire de $1 \mathrm{~m}$ environ au-dessus des tubes-guides, ce qui a permis d'obtenir un débit de dose d'ambiance inférieur à $0,1 \mathrm{mSv} / \mathrm{h}$.

\subsection{Opération de type $C$ (Gravelines 1 , Tricastin 2 et 4 )}

EdF ne disposait plus de jeux de tubes-guides neufs pour réaliser un échange standard; l'expérience de Fessenheim 2 et Tricastin 1 ayant mis en évidence la difficulté de mettre au point les stands chauds et la nécessité de s'affranchir des aléas entraînant un allongement des durées d'arrêt, il a été décidé de changer les broches en temps masqué par permutation des tubes-guides irradiés provenant d'autres tranches.

Une extension des locaux chauds du site de Tricastin a été réalisée. Cet atelier a été équipé de moyens de manutention et de trois stands chauds "simplifiés" construits par Framatome. Deux stands ont été affectés aux changements de broches, l'un fonctionnant en secours de l'autre. Le troisième stand a été utilisé aux changements de coupelles des vis de fixation de la bride intermédiaire reliant les deux parties de chaque tube-guide. En effet, les coupelles ont été souvent endommagées lors des opérations de manutention. Cette dernière opération "coûteuse en dose" sera améliorée dans le futur par l'adjonction d'une visserie adaptée par fixation de deux fausses brides protégeant les coupelles lors des opérations de manutention.

Le remplacement des tubes-guides de Tricastin 2 a été réalisé par une méthode dite "Aquarium" imaginée par les équipes de la centrale et développée par l'unité technique opérationnelle du Service de la production thermique. Afin de minimiser la durée de l'arrêt, le chantier tubes- 
guides est déplacé par dépose des internes supérieures dans un bidon appelé "Aquarium" rempli d'eau après manutention. Cette méthode permet de mener en parallèle les opérations de déchargement-rechargement du combustible et le remplacement des tubes-guides diminuant ainsi l'indisponibilité de la tranche. Cette procédure a permis de gagner 5 à 9 jours sur la durée de l'arrêt de tranche; en revanche, elle n'a pas apporté une baisse sensible de la dose intégrée par les opérateurs par rapport à une opération réalisée directement dans la piscine.

L'analyse du bilan dosimétrique de Gravelines 1 fait apparaître un coût élevé au poste manutention et transport des conteneurs de tubesguides (80 mSv). L'exploitant (unité technique opérationnelle) a amélioré la conception du château de transport en augmentant la hauteur ou le réhaussage pour obtenir une hauteur d'eau supplémentaire et en ajoutant deux coquilles extérieures en plomb afin de réaliser une protection biologique plus efficace.

La tranche 4 de Tricastin bénéficie du retour d'expérience de la tranche 2 , ce qui entraîne un coût dosimétrique diminué d'un facteur 2,5.

L'expérience montre qu'une importante partie des doses vient des opérations imprévues (par exemple manutentions mal préparées). La phase de préparation du travail est trop souvent confondue avec celles de l'organisation du chantier et la mise au point de l'outillage. En général, les opérations de manutention et de stockages intermédiaires des pièces radioactives demanderaient davantage d'études et de mises au point car, trop souvent, ces opérations sont considérées comme relevant du savoirfaire des chefs de travaux.

\subsection{Opération de type $D$ (usine à broches de Pierrelatte)}

Le transport des stands chauds et leur remontage étant à l'origine de nombreuses défaillances, il a été jugé préférable d'effectuer le remplacement des broches sur un seul site ; un atelier national sur le site de Pierrelatte (atelier B) a été créé à cet effet.

L'aménagement de l'atelier a été étudié pour exploiter quatre stands chauds Framatome et pour stocker les conteneurs de transport et de permutation de tubes-guides irradiants. Dans la cellule chaude, la barrette d'arrêt de la broche est détruite par étincellage, le dévissage de l'écrou est réalisé par une opération manuelle, un lavage des lamages est effectué avant contrôle de ceux-ci. La nouvelle broche est mise en place par un outillage semi-automatique, le serrage de l'écrou étant réalisé manuellement par une clé dynamométrique. La soudure d'arrêt de la barrette est faite automatiquement.

Le changement de broches se déroule par campagne, les tubesguides ne sont plus nécessairement affectés à la tranche d'origine. Le nombre de tubes-guides traités varie suivant leur provenance. Pour analyser le coût dosimétrique de l'exploitation de cet atelier, le critère dose par tube-guide paraît le plus intéressant, il est à rapprocher du coût dosimétrique du changement de broches sur le site : 


$\begin{array}{ll}\text { Fessenheim 1 : } & 9,48 \mathrm{mSv} \text { (sur site) } ; \\ \text { Fessenheim 2 } & 5,37 \mathrm{mSv} \text { (sur site) } \\ \text { Tricastin 1 } & 3,20 \mathrm{mSv} \text { (sur site) } \\ \text { Tricastin 4 } & 1,63 \mathrm{mSv} \text { (atelier B); } \\ \text { Dampierre 1 } & 1,62 \mathrm{mSv} \text { (atelier B) } \\ \text { Bugey 3 } & \text { 0,59 } \mathrm{mSv} \text { (atelier B). }\end{array}$

Les tubes-guides de Bugey 3 sont restés en stockage pendant une période supérieure à 90 jours, ce qui diminue l'activité due au cobalt 58 .

Pour connaître le coût dosimétrique de l'intervention sur les tubesguides d'une centrale, il est nécessaire d'ajouter au coût dosimétrique de l'atelier B celui de la manutention des tubes-guides en centrale. Ce dernier coût est significatif, comme le montrent les lignes suivantes, car le nombre de manutentions augmente :

$\begin{array}{ll}\text { Dampierre 1: } & 138 \mathrm{mSv} ; \\ \text { Bugey 3 } & 103 \mathrm{mSv} ; \\ \text { Gravelines 4 } & 187 \mathrm{mSv} ; \\ \text { Dampierre 2 } & 255 \mathrm{mSv} ; \\ \text { Gravelines 3 : } & 254 \mathrm{mSv} \text {; }\end{array}$

La dose reçue par les opérateurs lors de l'intervention sur la tranche 2 de Dampierre et sur la tranche 3 de Gravelines est supérieure à celle réalisée sur les autres tranches utilisant la même méthode (Aquarium ou conteneur). L'analyse de l'activité de l'eau du circuit primaire et du débit de dose d'ambiance au niveau de la piscine du réacteur met en évidence des valeurs supérieures à celles constatées sur d'autres tranches comparables. Une étude est en cours au département SécuritéRadioprotection-Environnement pour rechercher les causes de cette forte activité et pour engager des actions de réduction de l'activité des sources.

\section{CONCLUSIONS}

Le problème des broches rencontré sur les tranches REP $900 \mathrm{MW}$ est générique. C'est le revers de la standardisation, mais cet aspect est largement compensé par la possibilité de mettre en œuvre rapidement et sur l'ensemble des tranches des actions efficaces pour remédier aux anomalies (fig. 5).

La connaissance des premiers résultats dosimétriques a permis d'entreprendre des améliorations de la méthode de réparation afin de réduire le coût dosimétrique. On peut citer, parmi celles-ci :

- les améliorations de l'outillage, visant essentiellement à les simplifier : un outillage est d'autant plus fiable qu'il est simple ;

- l'organisation du chantier et la rigueur dans l'établissement des procédures, le retour d'expérience doit permettre de les parfaire ; la préparation des opérations de manutention, toujours très coûteuses en dose collective, mérite une attention particulière ;

- la réduction des sources radioactives en évitant de créer des effluents dont, l'activité et la forme chimique posent des problèmes de traitement, puis de stockage. 


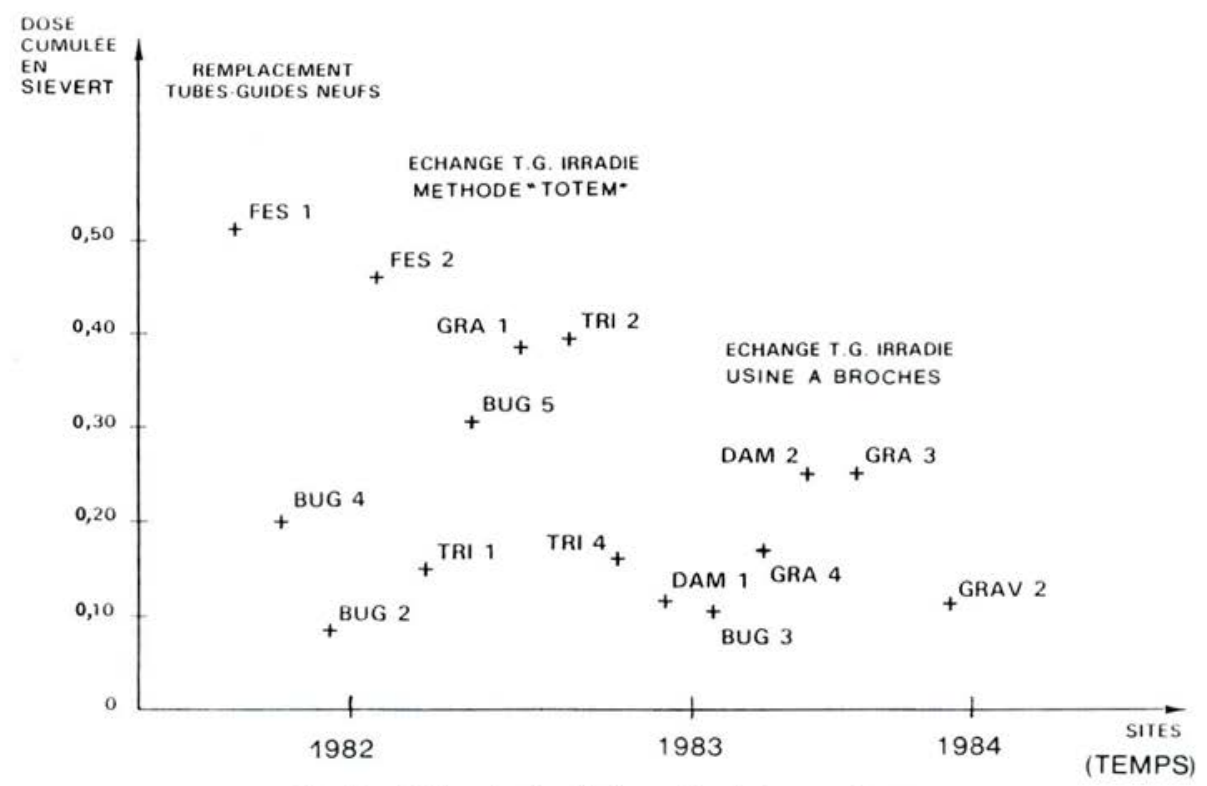

Fig. 5. - Bilan dosimétrique des interventions tubes-guides en centrale.

Une image du progrès dans le domaine de la réduction des doses peut être obtenue en suivant l'évolution de la dose collective totale reçue par les intervenants sur les tranches REP en service. Une synthèse des premiers résultats obtenus a permis d'évaluer le coût dosimétrique à une valeur moyenne de $2 \mathrm{~Sv}$ par tranche. Ce résultat se compare favorablement à ceux des centrales étrangères (3 à $3,5 \mathrm{~Sv}$ pour les Etats-Unis, et 2 à 3 Sv pour le Japon pour des centrales de mêmes tranches d'âge).

Le coût dosimétrique des interventions sur les broches des tubesguides représente $10 \%$ en moyenne de la dose collective annuelle d'une tranche, ce qui est un bon résultat, compte tenu que ce matériel est fortement irradiant.

Bien entendu, en fonction du vieillissement du parc, d'éventuels incidents conduisant à des réparations de matériels, la valeur de 2 Sv/an risque d'augmenter. C'est pourquoi, la réduction des doses reçues par le personnel travaillant dans les centrales nucléaires est un souci permanent d'Electricité de France. 